\title{
Improving Freehand Placement for Grasping Virtual Objects via Dual View Visual Feedback in Mixed Reality
}

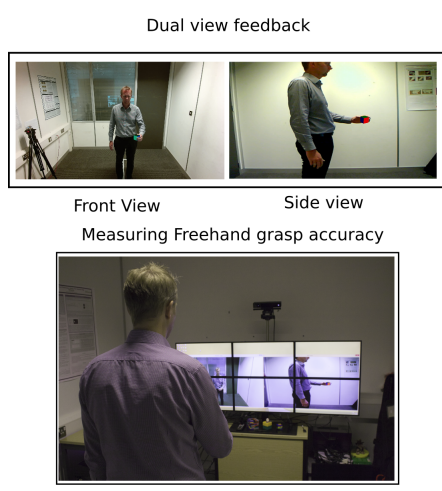

(a)
Experimental Environment

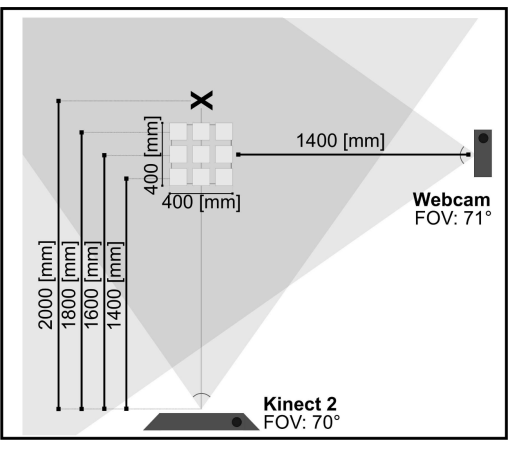

(b)
Grasp Mode

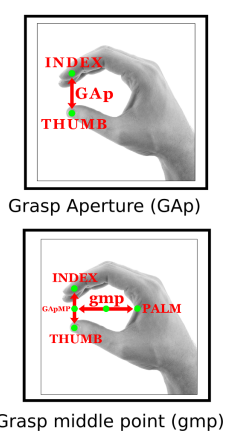

(c)

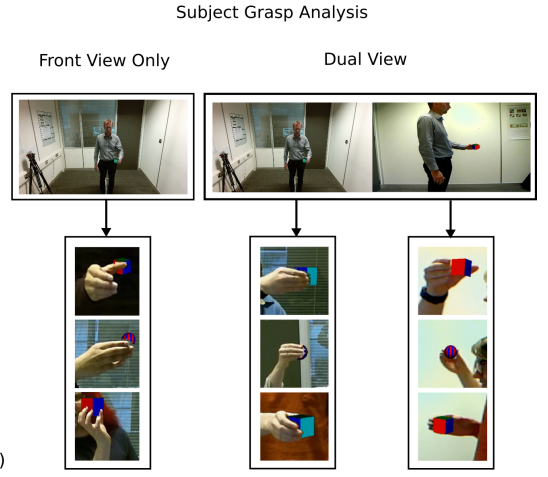

(d)

Figure 1: Assessment of dual view visual feedback for freehand grasping in mixed reality

\begin{abstract}
This paper present a first study into the use of dual view visual feedback in an exocentric MR environment for assisting freehand grasping of virtual objects. A recent study has highlighted the problems associated with user errors in freehand grasping, via an analysis of virtual object type, location and size. This work present an extension to this evaluation, where the same 30 participants were recruited for two experiments (one assessing object size and the second object position). We report on results following the same protocol of the aforementioned study in a laboratory controlled environment using a dual view visual feedback method. We present a comprehensive statistical analysis of the comparison between a single view and dual view feedback method alongside user evaluation using the System Usability Scale (SUS). The results presented clearly show that the dual view visual feedback significantly increases user z placement accuracy and improves grasp placement in the $\mathrm{x}$ and $\mathrm{y}$ axes, however completion time was significantly higher. No variation or improvement was found in user grasp aperture using dual view visual feedback for changes in object size/position. We present conclusions on SUS and offer directions forward for interaction feedback.
\end{abstract}

Keywords: Grasping, Freehand Interaction, Natural Hand Interaction, Human Performance Measurement, Visual Feedback, Dual View Feedback, Mixed Reality

Concepts: -Computing methodologies $\rightarrow$ Mixed / augmented reality; •Human-centered computing $\rightarrow$ User studies; Interaction techniques; User centered design; Usability testing;

\section{Introduction}

Freehand grasping, where a user manually interacts with virtual objects, is one of the most desirable forms of natural interaction for Mixed, Augmented or Virtual reality (MR/AR,VR). While this is the case, the the many challenges faced for natural freehand grasping and the objective assessment of human errors in freehand virtual object grasping has largely been unexplored. The recent work of Al-Kalbani et al. [2016] has sought to address this and developing on the studies of [MacKenzie and Iberall 1994] for real object grasping, quantified the errors introduced when users aim to manu- ally grasp virtual objects in an exocentric MR environment. While the work of Al-Kalbani et al. [2016] presents an initial study into the freehand grasp problems and proposes measures for estimating the errors introduced by users in freehand grasping, it illustrates a significant problem in user grasp accuracy, namely how users often fail to accurately estimate the correct depth location of virtual objects and how the grasp aperture does not change linearly to the changes in virtual object size.

Within this work we present an extension to the evaluation of AlKalbani et al. [2016], notably in assessing to what extent the grasp displacement can be improved by providing additional visual feedback to the users. We illustrate how improvements can be made to user depth estimation thus overcoming the underestimation reported by Al-Kalbani et al. [2016]. We also show that the common errors when users grasp virtual objects in an MR scene can be largely mitigated using a dual view exocentric MR environment. Size, Shape and object position are compared using dual view against a single view and the System Usability Score (SUS) is applied to assess the user response and preference.

The paper is structured as follows: Section 2 details the theories of feedback in MR, comparing the use of multi-modal and visual feedback methods and illustrating that dual view feedback ins novel to this work. Section 3 then presents the methodology for our experiments, replicating the experimental controls of AlKalbani et al. [2016]. Sections 4 and 5 present the two experiments conducted and detail findings for dual view visual feedback in comparison to single view feedback. Section 6 concludes the work stating the key findings and routes for future analysis.

\section{Related Work}

\subsection{Feedback in MR}

Feedback is defined in a general context as the process in which the impact of an action is returned to improve or correct the next action. Absence of feedback can lead to poorer performance in MR, AR and VR environments [Maria et al. 2015] and use of suitable feedback can lead to direct improvements in user performance [Pitts et al. 2012].

Feedback modalities vary, with visual, audio, haptic, tactile and force feedback commonly used within MR. Multimodal feedback is also widely used, and aims to improve user performance through 
the integration of two or more feedback modalities, thus giving 142 users a higher sense of presence in relation to virtual elements in ${ }_{143}$ MR environments.

\subsubsection{Multimodal Feedback}

Due to the potential advantages of multimodal feedback, it is an active research field with studies aiming to assess the ideal combination of modes for a given environment. Duff et al. [2010b; 2010a] presented a mixed reality system for stroke rehabilitation with multimodal visual and aural feedback. Even though the number of users was limited to 3 in the usability evaluation, promising results were shown for reaching movements. Pacchierotti et al [2012] compared force feedback modalities to visual feedback in two finger grasping of virtual objects. Findings in their work showed that force feedback improves the accuracy of a user's grasp.

Distance perception for moving objects in a virtual environment using audio, visual and audio-visual feedback was studied by Rebillat et al. [2012]. Large immersive displays in two orientations were used in their work for providing immersive visual feedback, however quantative analysis of their feedback was not explored. Prachyabrued and Borst [2014] also investigated feedback for virtual grasping, again using visual and audio feedback, and presented promising recommendations for mitigating the problems of real hand penetration of virtual objects. Pitts et al. [2012] investigated the interaction of visual and haptic feedback in automotive touchscreen simulation. Their results have shown that using haptic feedback alongside visual feedback improves performance and reduces glance time.

Combinations of real time multimodal feedback (i.e visual, audio, haptic, tactile and force) in VR, AR and MR training systems and simulations is the subject of much research in the medical domain [Duff et al. 2010b; Duff et al. 2010a; Baran et al. 2011; Sutherland et al. 2013; Coles et al. 2011; Jia et al. 2013; Horeman et al. 2012]. Promising results in terms of functionality, adaptive integration of feedback modalities and novel contributions of these medical systems have been shown. Again system evaluation is generally limited with subjective and informal analysis commonly presented. Additional methods for multimodal feedback in a AR rehabilitation systems have applied projection mapping techniques, notably Vieira et al. [2015]. Their system used projection mapping techniques for visual feedback, and added haptics and audio feedback modalities to increase sources of awareness in a rehabilitation task. Even though it was claimed that this system showed promising results in guiding motion in rehabilitation tasks through multimodal feedback, a formal evaluation study has not been implemented. It is evident from the literature that methods combining different feedback modalities with visual feedback do benefit performance in interaction. However this can be limited where freehand interaction, without any wearable device, is required and visual feedback is commonly used alone. More recently methods to improve the level of visual feedback for users in freehand interaction and extending this into multiple view methods, are being developed [Johnsen et al. 2014].

\subsubsection{Multiple Views in Visual Feedback}

Visual feedback in MR and AR is considered to be a conventional type of feedback [Prattichizzo et al. 2012] that is widely used. Dey and Sandor [2010; 2014] used visual feedback on handheld AR displays to compare different visualizations of occluded virtual objects in outdoor environments. Key insights and recommendations were offered regarding the impact of size and resolution of handheld AR displays, depth perception in outdoor environments and the effect of motion parallex on handheld AR systems. Two depth cameras and visual feedback through an optically transparent LCD screen was utilised by Lee et al. [2013] to present 3D spatial interaction with virtual objects on a desktop setup. A novel concept in extending traditional 2D desktop interaction to spatial 3D manipulation was presented in their work. However, no objective analysis was provided to asses the system developed. Chang et al. [2012] simultaneously compared Microsoft's Kinect with an OptiTrack motion capture sensor, and used visual feedback to assess rehabilitation performance and progress of patients in a game setup. Even though quantitative analysis was provided in their study, only two participants were included in the evaluation process, and the impact of feedback on rehabilitation was not investigated. Johnsen et al. [2014] developed a virtual pet training MR system through gesture and speech recognition to reduce child obesity by using physical activity of participants as input. Visual feedback through a standard LCD monitor in their work was divided into three regions occupying varying sizes of the overall monitor area, with each region presenting different information to users. A comprehensive analysis of the system was presented, however, the impact of dividing the visual feedback into three regions was not investigated, and information presented in all three regions of the visual feedback only showed synthetic virtual components and not participants in the real environment. Ha et al. [2014] combined two depth cameras and used visual feedback through an HMD to allow freehand manipulation of 3D virtual objects. Different rendering methods of virtual objects were tested and analysed to improve depth perception. However, their work was only limited to testing different visualization methods and not views. Projection mapping techniques [Wilson and Benko 2010; Hilliges et al. 2012; Benko et al. 2014; Jones et al. 2014] have also been the subject of much research. With the aid of multiple projectors and depth cameras, these techniques transform a real world space into a unique augmented experience for users. Even though projection system use visual feedback, they are usually presented as prototypes that are informally assessed, and are mainly focused on user experience.

While the literature discussed offers evidence for the wide use and benefits of visual feedback in MR environments, using different visual feedback views to mitigate the problems of spatial grasp placement in the $\mathrm{x}, \mathrm{y}$ and $\mathrm{z}$ axes has not been explored. Moreover, the usability of dual view visual feedback is unclear, and this novel visual feedback presented in this paper for freehand grasping in an MR context has not yet been explored.

\section{Experiment Design}

Following the same protocol, guidelines and design, this study performs a replication of the experiment presented in [Al-Kalbani et al. 2016] with the addition of a second camera, providing a dual visual feedback (see Fig. 1a and Fig. 1b). We also focus on the last phase of a grasp, as defined in the work of [MacKenzie and Iberall 1994], and assess the medium wrap grasp [Bullock et al. 2013; Feix et al. 2014]. We use the Grasp Aperture (GAp) and Grasp Displacement (GDisp) metrics defined in equations 1 and 2 from the grasp model presented in [Al-Kalbani et al. 2016] to measure grasp placement.

$$
G A p=\sqrt{\left(P_{x}-B_{x}\right)^{2}+\left(P_{y}-B_{y}\right)^{2}+\left(P_{z}-B_{z}\right)^{2}}
$$

$$
g m p=\left(\frac{G A p M P_{x}+p^{2} m_{x}}{2}, \frac{G A p M P_{y}+\text { palm }_{y}}{2}, \frac{G A p M P_{z}+p a l m_{z}}{2}\right)
$$

Where GAp is the distance between the index and thumb fingers in the $\mathrm{x}, \mathrm{y}$ and $\mathrm{z}$ axes, and GDisp is the distance between the grasp middle point $(\mathrm{gmp})$ and the object middle point (omp) in the $\mathrm{x}, \mathrm{y}$ and $\mathrm{z}$ axes (see Fig. 1c).

To directly follow the methodology of Al-Kalbani et al [2016] we compare the influence of object size, shape and position (in $\mathrm{x}, \mathrm{y}$ and $z$ space) on grasp accuracy using the dual visual feedback (see Fig. 1d) on simple abstract shapes. Table 1 displays the conditions of the two experiments. 
Table 1: Experiment conditions, where $x$ is measured from the centre of the sensor, $y$ from ground and $z$ from sensor

\begin{tabular}{|c|c|c|c|c|}
\hline \multicolumn{5}{|c|}{ Object Size Experiment } \\
\hline Condition & \multicolumn{4}{|c|}{ Levels } \\
\hline Size & \multicolumn{4}{|c|}{$40 \mathrm{~mm}, 50 \mathrm{~mm}, 60 \mathrm{~mm}, 70 \mathrm{~mm}, 80 \mathrm{~mm}, 100 \mathrm{~mm}$} \\
\hline Object Type & \multicolumn{4}{|c|}{ Cube and Sphere } \\
\hline \multicolumn{5}{|c|}{ Object Position Experiment } \\
\hline Condition & \multicolumn{4}{|c|}{ Levels } \\
\hline \multirow[t]{4}{*}{ Position (x, y) } & & LEFT & MIDDLE & RIGHT \\
\hline & TOP & $-40,1290$ & 0,1290 & 40,1290 \\
\hline & CENTRE & $-40,1250$ & 0,1250 & 40,1250 \\
\hline & BOTTOM & $-40,1210$ & 0,1210 & 40,1210 \\
\hline Object Type & \multicolumn{4}{|c|}{ Cube and Sphere } \\
\hline
\end{tabular}

The 9 positions were repeated in each z plane $(1400 \mathrm{~mm}, 1600 \mathrm{~mm}$ and $1800 \mathrm{~mm})$ as shown in Fig. $1 b$.

\subsection{Participants}

The same 30 participants that took part in [Al-Kalbani et al. 2016] were recruited from a population of university students and staff members. Participants completed a standardized consent form, were not compensated, and all data collected was anonymised. Visual acuity was measured using a Snellen chart, and each participant was required to pass an Ishihara test to exclude for colour blindness. No participants suffering from colour blindness and/or with visual acuity of $<0.80$ were included in the analysis. Height, arm length and hand size of all participants were also measured prior to each experiment, to ensure that aspects of the experimental design are within the biomechanical reach of participants. All participants were right handed [Oldfield 1971].

Participants ranged in age from 19 to $62(M=30.43$, SD $=9.78)$, in arm length from $480 \mathrm{~mm}$ to $660 \mathrm{~mm}(M=552.40, \mathrm{SD}=43.80)$, in hand size from $160 \mathrm{~mm}$ to $200 \mathrm{~mm}(M=186.80, \mathrm{SD}=10.40)$, in height from $1570 \mathrm{~mm}$ to $1950 \mathrm{~mm}(M=1744.00, \mathrm{SD}=90.00)$ and 6 were female and 24 male. Taking into account balance in hand size, arm length, gender, age and height, we have separated participants into two groups of 15 for the two experiments.

\subsection{System Architecture}

The system developed integrated the use of a Microsoft Kinect 2 (FOV: $70.0^{\circ}$ ), a (HD) video camera, a Live! Cam Optia Pro HD webcam $^{1}$ (FOV: $71.0^{\circ}$ ) and a SyncMasterX6 ${ }^{2}$ feedback monitor. We opted for using a HD webcam as a second view feedback camera as two Kinect sensors on one PC is not yet supported [Benko et al. 2014]. FOV of the Kinect and webcam were different to a small degree, however, the full interaction space was visible on both views. The experiments were developed in $\mathrm{C}++$ using Kinect SDK. Autodesk Maya ${ }^{3}$ was used for modelling 3D objects, open computer vision library OpenC $\mathrm{CV}^{4}$ for video processing and open graphics library OpenGL ${ }^{5}$ for real time reading, loading and texturing of three dimensional (3D) virtual objects.

The physical configuration of the sensor strictly followed the recommendations of Kinect's 2 manufacturers ${ }^{6}$. Participants stood $2000 \mathrm{~mm}$ away from the sensor under controlled and constant lighting conditions, the sensor was placed at a height of $1800 \mathrm{~mm}$ and tilted at an angle of $13.78^{\circ}$ to show the interaction space around participants and to eliminate any significant self occlusion prob-

\footnotetext{
${ }^{1}$ http://support.creative.com/kb/ShowArticle.aspx?sid=10859

${ }^{2}$ http://www.samsung.com/us/support/owners/product/MD230X6

${ }^{3}$ http://www.autodesk.com/products/maya/overview

${ }^{4}$ http://opencv.org/

${ }^{5}$ http://www.opengl.org/

${ }^{6}$ http://support.xbox.com/en-GB/xbox-360/kinect/kinect-sensor-setup
}

lems (see Fig. 1).

Participants stood $1400 \mathrm{~mm}$ away from the side view webcam, placed to the left hand side and at the same height as the Centre Middle position $(1250 \mathrm{~mm})$ presented to participants in Object Position Experiment (see Table. 1). To ensure all objects in varying positions in the Object Position are visible to participants on the feedback monitor. As the distance to the webcam was smaller to the one from the Kinect sensor, 3D virtual objects were computed to be larger in OpenGL to reflect an accurate representation of the closer distance to participants.

We have chosen to place the second view visual feedback to the side of participants as results in [Al-Kalbani et al. 2016] have shown that Grasp Displacement in the $\mathrm{x}$ axis was user dependant and was influenced by the dominant hand of users, not the feedback method. On the other hand, Grasp Displacement in the y axis was influenced by the feedback method, thus spatial placement of the hand in the $y$ axis was affected by the visual feedback method used. Moreover, highest Grasp displacement was found in the $\mathrm{z}$ axis due to using single view visual feedback, thus we used a side view as a second visual feedback method to show the $y$ and $\mathrm{z}$ axes, the two axes that were directly affected by the feedback method used in [AlKalbani et al. 2016]. Moreover, high grasp variation was found in [Al-Kalbani et al. 2016], meaning that participants used different grasp types to the one they were instructed to use in the test. This behaviour was attributed to participants trying to visualise their full hand. Adding a side view allows participants to visualise all parts of their hand without the need to adapt their grasp type.

The feedback monitor was split into two equally sized side by side windows, showing the frontal view feedback from the sensor on the left hand side window, and the side view feedback from the webcam on right hand size window (see 1a). Positions of the windows on the feedback monitor were unchanged throughout the study. However, participants were asked to comment on the positions of the windows and their influence on their performance in the subjective analysis after the experiments.

\subsection{Experimental Protocol}

Participants were naive to the purposes of the experiments, but their level of experience in MR systems ranged from novice to expert. Participants stood $2000 \mathrm{~mm}$ away from the monitor (size: 62in $\times$ 30in, resolution: $5760 \times 2160$ ), displaying a composited dual-view real time mirrored scene overlaying virtual objects with the video feed obtained from both the Kinect and the Live! Cam. Grasping parameters ( $G A p, G D i s p)$ are measured from the sensor, not to test biomechanics of the hand but to quantify errors in spatial positioning and aperture estimation.

Participants underwent initial training of the medium wrap grasp on real and virtual objects, and were given time to familiarise themselves with the side view visual feedback concept. The test coordinator explained the procedure between each block of tests (i.e cube and sphere), and participants were allowed to rest before presentation of every object. Each experiment was formed of a 5 minutes training/instruction session, 10 minutes of grasping a cuboid object, 5 minutes break and 10 minutes of grasping a spherical object. During the experiment all participants were instructed to verbally inform the test coordinator that they are satisfied with the grasp they have performed on both feedback views (frontal and side), and maintain the grasp for 5 seconds while the measurements are stored. After completing the test, participants were asked to fill in a usability questionnaire and a set of questions regarding their interaction with the system. The usability of the system was evaluated by a user satisfaction test based on the System Usability Scale (SUS) [Brooke 1996]. This questionnaire consists of 10 items, which were evaluated by using a Likert scale ranging from 1 (strongly disagree) to 5 (strongly agree). Through feedback from this questionnaire, we were able to evaluate the ease of use and usability of this new con- 
figuration of the system.

In order to further assess interaction strategies and behaviour pro- 36 tocols by participants while using the system they were asked to ${ }_{367}$ answer a set of 6 close-ended questions. These questions were pre- 368 sented as a post test questionnaire and participants commented on 369 anything they considered related to the questions. Questions were: 370 1) Which screen did you look at first? 2) Which screen did you 371 depend on the most? 3) Which view did you find to be more impor- ${ }_{372}$ tant? 4) If used again, would you rather use two feedback views or ${ }_{373}$ just one (frontal view)? 5) Did you use the dual view in a specific 374 order? 6) Do you think changing positions of both feedback screens 375 would make a difference in performance?

\subsection{Statistical Analysis}

Kruskal Wallis H test [1952] is used for analysis over the ANOVA ${ }_{379}$ test as our data did not follow a normal distribution [Field 2012]. A 380 post-hoc test for multiple comparisons using Dunn Test with Bonferroni correction [Dunn 1961] is preferred to a Mann-Whitney U post hoc test [Iman and J. 1983], and is used for statistically significant results of the Kruskal Wallis $\mathrm{H}$ test, to check for statistical differences using pairwise comparisons between groups of independent variables.

In our results and analysis, we focus on the differences between visual feedback methods, and only compare the grasp accuracy metrics (GAp and GDisp) between the two feedback views (single view and dual view) as whole sets, thus we do not analyse all the levels in our conditions (sizes, positions and object type), as this level of detailed analyses was presented in the work of [Al-Kalbani et al. 2016].

\section{Object Size Experiment}

We used a $2 \times 6$ within-subjects design, with two primary conditions: object size and object type (see Table 1). All 15 participants took part in both conditions. Every permutation for both object types was randomly presented to participants to exclude potential learning effects. In total, each participant completed 6 (sizes) $\times 5$ (repetitions) $\times 2$ (objects $)=60$ trials and 900 grasps $(60$ trials $\times 15$ participants). Each static grasp of every participant was recorded for 5 seconds (75 frames), leading to collecting 67500 raw data points (900 grasps $\times 75$ frames).

Hypothesis: We test the null hypotheses that using dual visual feedback in grasping virtual objects that change in size has no effect on a) grasp aperture and b) grasp displacement.

\subsection{Procedure}

Participants were instructed to accurately match their grasp aperture to the size and position of the virtual object in the shortest time possible on both feedback views. During the experiment, an object (cube or sphere) appeared on the feedback monitor, in 6 different sizes (see Table 1). Objects were positioned $1600 \mathrm{~mm}$ away from the sensor and $400 \mathrm{~mm}$ away from participants ( $\mathrm{z}$ ), at a height of $1250 \mathrm{~mm}(\mathrm{y})$ and at the zero $(\mathrm{x})$ point on the sensor. This position was constant throughout the experiment.

\subsection{Results and Analysis}

\subsubsection{Results - Grasp Aperture (GAp)}

No statistically significant difference was found in Grasp Aperture $(G A p)$ between the two visual feedback methods (single view and dual view) in grasping cubes $\left(\chi^{2}(1)=5.06, p>0.01\right)$. For spheres, statistically significant difference was found in Grasp Aperture $(G A p)$ between the two visual feedback methods $\left(\chi^{2}(1)=1270.90\right.$, $p<0.01)$.

In order to understand the practical significance of the multiple comparisons in our post-hoc analysis, Cohen's $d$ [Cohen 1992] effect size for independent t-tests is calculated. A negligible effect size $(d<0.20)$ for cubes, and a small effect size for spheres

$(d<0.30)$ were found.

This shows that using dual view visual feedback for freehand medium wrap grasping still presents comparable results to previous work on single view visual feedback [Al-Kalbani et al. 2016]. Object size that presented the lowest mean difference between $G A p$ and cubes $(80 \mathrm{~mm})$ was the same to the one reported in [Al-Kalbani et al. 2016], but different for spheres $(80 \mathrm{~mm})$ as $70 \mathrm{~mm}$ was the size reported in [Al-Kalbani et al. 2016]. This indicates that object sizes between $70 \mathrm{~mm}$ and $80 \mathrm{~mm}$ present most accuracy in GAp regardless of the feedback method used.

Even though the difference between mean $G A p$ across all sizes of cubes and spheres was negligible $(0.07 \mathrm{~mm})$ in this study, it still contradicts findings in the single view visual feedback study. As we report lower mean $G A p$ across all sizes was found for cubes than spheres, the opposite to our findings were reported for single view visual feedback.

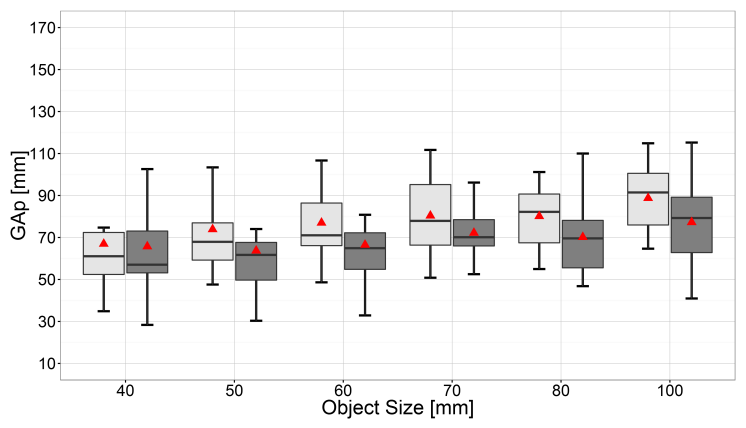

(a)

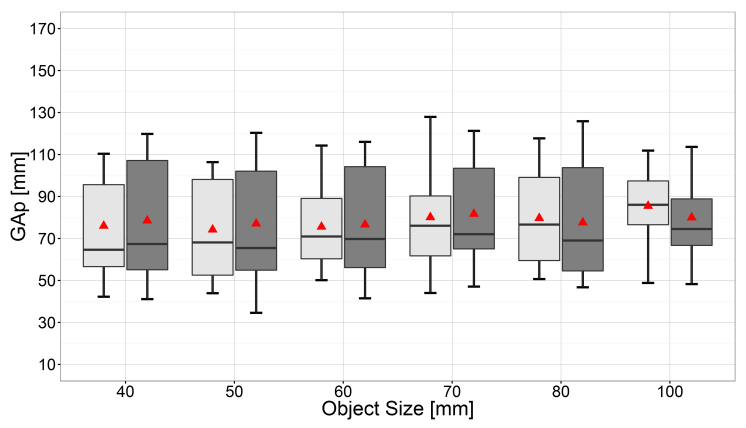

(b)

Figure 2: GAp for different object sizes in the $1600 \mathrm{~mm}$ z plane in the Object Size Experiment. Light grey boxplots show cubes, and dark grey boxplots show spheres. Red triangles on boxplots indicate the mean GAp across all participants for each size. Whiskers represent the highest and lowest values within 1.5 times the interquartile range from the lower and upper quartiles: (a) Single view visual feedback from [Al-Kalbani et al. 2016] (b) Dual view visual feedback

Participants maintained their behaviour in matching their GAp to object size with the addition of side view visual feedback. For both objects, participants overestimated object size up until the size that had the lowest mean difference between $G A p$ and object size ( $80 \mathrm{~mm}$ for cube and sphere). In addition, both objects showed that with the $100 \mathrm{~mm}$ size, participants underestimated its size by a mean of $-14.51 \mathrm{~mm}$ for cubes $(\mathrm{SD}=24.92)$, and $-20.02 \mathrm{~mm}$ for spheres $(\mathrm{SD}=28.59)$. This behaviour was compatible between one and dual view visual feedback.

Fig. $2 \mathrm{~b}$ further shows the mean $G A p$ range across all sizes of both objects. Even though this range is smaller than the range of $G A p$ reported for single view visual feedback (see Fig. 2a), it still shows that, given object sizes ranged from $40 \mathrm{~mm}$ to $100 \mathrm{~mm}$, responsive- 
ness of participants in terms of accurately matching GAp to object size is constrained between $60 \mathrm{~mm}$ and $80 \mathrm{~mm}$, regardless of the feedback method used. This finding was surprising in this work as participants had additional side view visual feedback, that clearly showed their thumb and index fingers, to accurately match their $G A p$ to object size. Even though significant differences were found between the two visual feedback methods, dual visual feedback did not show any improvements over single view feedback in $G A p$ matching to object size, and no direct linear relationship between object size and $G A p$ as in real objects was found. As statistically significant results were found for the feedback method condition, the null hypothesis that dual visual feedback in grasping virtual objects that change in size does not have an effect on GAp is rejected.

\subsubsection{Results - Grasp Displacement (GDisp)}

Statistically significant difference was found in Grasp Displacement in the $\mathrm{x}$ axis $\left(G D i s p_{x}\right)$ between the two visual feedback methods in grasping cubes $\left(\chi^{2}(1)=2875.70, p<0.01\right)$. For spheres, no statistically significant difference was found in GDis $p_{x}$ between the two visual feedback methods $\left(\chi^{2}(1)=4.20, p>0.01\right)$. A small effect for cubes $(d>0.30)$, and a negligible effect for spheres were found $(d<0.20)$.

Similar to single view visual feedback, positive GDis $p_{x}$ was present for both objects. This positive GDisp $p_{x}$ is expected, as all participants were right handed, and the Grasp Middle Point ( $\mathrm{gmp}$ ) was computed on the right hand side of virtual objects. Mean $G D i s p_{x}$ was lower for both objects using dual view visual feedback $(19.33 \mathrm{~mm}(\mathrm{SD}=21.40)$ for cubes, and $31.01 \mathrm{~mm}(\mathrm{SD}=20.28)$ for spheres) than single view visual feedback $(28.01 \mathrm{~mm}(\mathrm{SD}=14.08)$ for cubes and $31.52(\mathrm{SD}=14.68)$ for spheres). This shows that adding side view visual feedback significantly improves the $g m p$ spatial positioning in the $\mathrm{x}$ axis and reduces GDis $p_{x}$ from the centre of virtual objects.

Similarity in $g m p$ placement on the $\mathrm{x}$ axis was reported for single view visual feedback as shown by the range of clusters on the $\mathrm{x}$ axis in Fig. 3a. Range of the mean GDisp $p_{x}$ across all sizes of cubes and spheres was lower using dual view visual feedback (see Fig. 3b). No significant difference was found in Grasp Displacement in the $\mathrm{y}$ axis $\left(G D i s p_{y}\right)$ between the two feedback methods in grasping cubes $\left(\chi^{2}(1)=5.89, p>0.01\right)$. For spheres, statistically significant difference was found in GDispy between the two feedback methods $\left(\chi^{2}(1)=2551.50, p<0.01\right)$. A negligible effect for cubes 465 $(d<0.20)$, and a small effect for spheres were found $(d<0.50) . \quad{ }_{466}$ Negative GDisp $y$ was present for both objects. This reveals that par- ${ }_{467}$ ticipants placed their gmp below the Object Middle Point (omp), a ${ }_{468}$ behaviour that was also present in single view feedback and is po- 469 tentially attributed to participants trying to show parts of the objects 470 presented to them on the feedback monitor, a strategy that reassured 471 participants that they have grasped the virtual object.

Mean GDisp y was lower for both objects using dual view visual 473 feedback $(-12.13 \mathrm{~mm}$ (SD = 11.10) for cubes, and $-4.85 \mathrm{~mm}$ (SD 474 $=12.02)$ for spheres) than single view visual feedback $(-12.37 \mathrm{~mm} \quad 475$ $(\mathrm{SD}=11.94)$ for cubes, and $-9.84 \mathrm{~mm}(\mathrm{SD}=12.51)$ for spheres), 476 this shows that dual view visual feedback significantly improves the 477 gmp spatial positioning in the y axis by reducing GDisp $y$ from the ${ }_{478}$ centre of virtual objects. Similar to GDis $p_{x}, g m p$ placement across 479 participants on the y axis was comparable across object sizes as 480 shown by the range of clusters in Fig. 3b. This consistency in gmp 481 placement on the y axis was present using one visual feedback (see ${ }^{482}$ Fig. 3a).

Mean GDisp $p_{x}$ and GDisp $p_{y}$ for each object size in both objects have ${ }_{484}$ shown that placement of $g m p$ shifted towards the 0 origin of the ${ }_{485}$ $\mathrm{x}$ and $\mathrm{y}$ axis as shown in Fig. 3b, this indicates that even though 486 GDisp $p_{x}$ and GDisp $p_{y}$ are still existent with the use of dual view ${ }_{487}$ visual feedback, the displacement is reduced and is closer to the ${ }_{488}$ origin of the virtual object than it was with using single view vi- 489
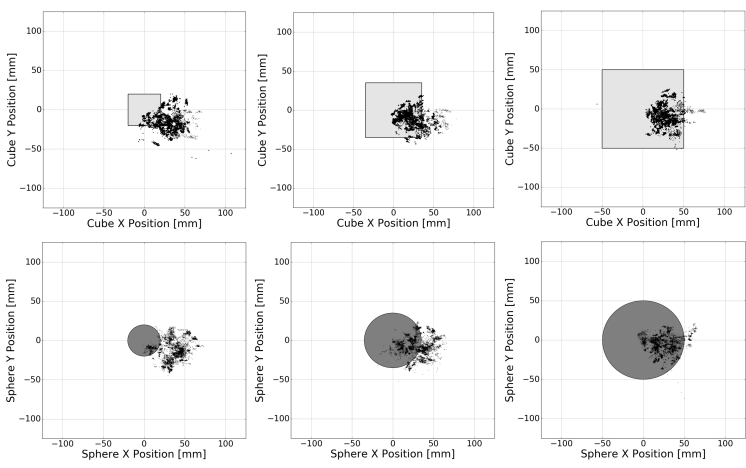

(a)
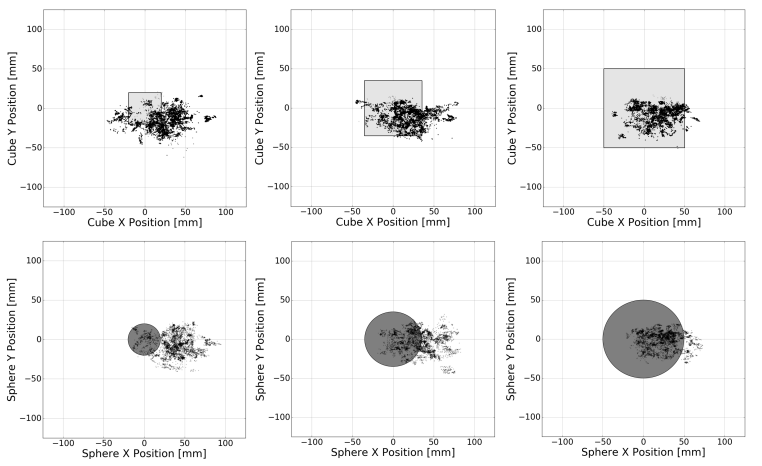

(b)

Figure 3: gmp placement (black clusters) in the $x$ and $y$ axes of all participants in the Object Size Experiment presenting 3 sizes (40mm, $70 \mathrm{~mm}, 100 \mathrm{~mm}$ ) for cubes (top row) spheres (bottom row): (a) Single view visual feedback from [Al-Kalbani et al. 2016] (b) Dual view visual feedback

sual feedback. Moreover, SD differences of GDis $p_{x}$ and GDisp means within object sizes between cubes and spheres were comparable, indicating that contact of $g m p$ with the surface of the object was reflective of size growth of objects rather than movements by participants. This behaviour was compatible between one and dual view visual feedback, and it shows that even though dual view visual feedback reduces GDis $p_{x}$ and GDis $p_{y}$ and moves participants closer to the centroid of virtual objects in the $\mathrm{x}$ and $\mathrm{y}$ axis, participants remain consistent in their spatial $g m p$ placement regardless of changes in object size. This consistency is expected as object position was unchanged throughout this experiment.

Statistically significant difference was found in Grasp Displacement in the $\mathrm{z}$ axis $\left(\right.$ GDisp $\left._{z}\right)$ between the two feedback methods in grasping cubes $\left(\chi^{2}(1)=2420.30, p<0.01\right)$ and spheres $\left(\chi^{2}(1)\right.$ $=5752.40, p<0.01)$. Medium effects for cubes and spheres $(d<0.80)$ were found.

GDisp $p_{z}$ presented the highest displacement out of all three axis with single view visual feedback in [Al-Kalbani et al. 2016], and in this study we test if dual view visual feedback can mitigate high GDisp and aid in achieving accurate depth positioning in MR. In our work, the terms underestimation and overestimation are opposite to those of depth perception, hence in this study, depth refers to the distance from the feedback monitor and not the user as in depth perception studies. Negative mean GDisp $p_{z}$ was found for both objects across all sizes, this indicates that majority of participants underestimated the $\mathrm{z}$ position of $o m p$ by placing their $g m p$ in front of the $o m p$ for all sizes. Overestimation of $\mathrm{z}$ position was also present, but not as frequent as underestimation, as $54 \%$ of the data showed underestimation, while overestimation was present in $45 \%$ of the data. However, the difference between overestimation and underestimation is smaller and distributed in a more balanced manner when us- 


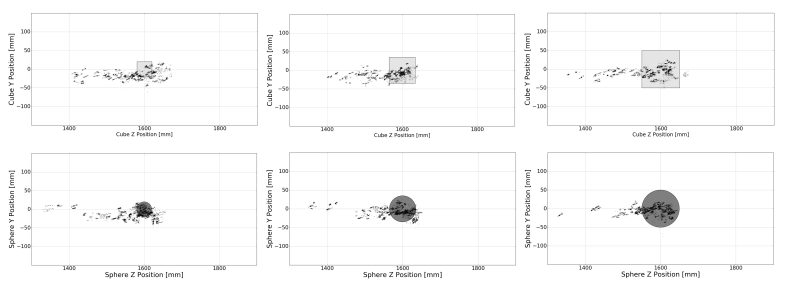

(a)

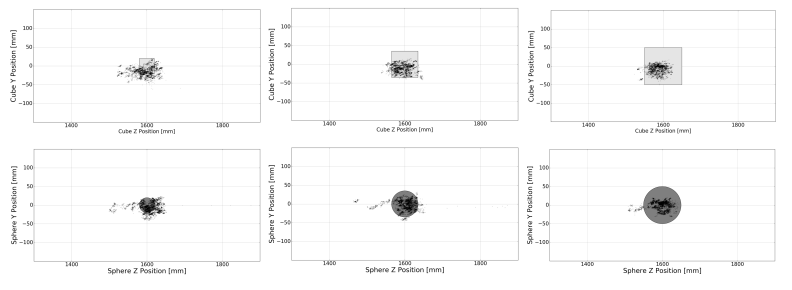

(b)

Figure 4: gmp placement (black clusters) in the z axis of all participants in the Object Size Experiment presenting 3 sizes (40mm, 70mm, 100mm) for cubes (top row) spheres (bottom row):

(a) Single view visual feedback (b) Dual view visual feedback

ing dual view visual feedback than single view visual feedback, as underestimation was reported to be present in $67 \%$ of the data, and overestimation was present in $33 \%$ of the data using single view visual feedback. Position of gmp in the $\mathrm{z}$ axis was comparable across all sizes for both objects, and more clustered in the centre of objects as shown by Fig. 4b. This is attributed to the more balanced distribution of $\mathrm{z}$ position overestimation and underestimation caused by dual view visual feedback.

Mean GDisp $p_{z}$ range was lower using dual view visual feedback as shown in Fig. 4b than one visual feedback. Mean GDis $p_{z}$ was lower for both objects using dual view visual feedback $(-8.96 \mathrm{~mm}(\mathrm{SD}=$ 22.56) for cubes and $1.20 \mathrm{~mm}(\mathrm{SD}=27.55)$ for spheres) than single view visual feedback $(-38.39 \mathrm{~mm}(\mathrm{SD}=61.67)$ for cubes and $-29.87 \mathrm{~mm}(\mathrm{SD}=60.51)$ for spheres). This shows that dual view visual feedback significantly reduces $G D i s p_{z}$, and improves gmp spatial positioning in the $\mathrm{z}$ axis by reducing GDisp $p_{z}$ from the centre of virtual objects. Moreover, dual view visual feedback reduced deviation in GDisp $p_{z}$ as shown by the SD values when compared to reported values of single view visual feedback (see Fig. 4a). As statistically significant results were found for the feedback method condition, the null hypothesis that dual visual feedback in grasping virtual objects that change in size does not have an effect on GDisp is rejected. As dual visual feedback has a significant effect on GDisp in all axes (x, $\mathrm{y}$ and $\mathrm{z}$ ).

\subsubsection{Results - Completion Time}

Statistically significant difference in completion time between the 577 two feedback methods was found for cubes $\left(\chi^{2}(1)=18863, p<578\right.$ $0.01)$ and spheres $\left(\chi^{2}(1)=16551, p<0.01\right)$.

Statistically significant difference in completion time between the two feedback methods shows that adding a second view camera for visual feedback significantly increases completion time, as the overall completion time across all sizes was $7.65 \mathrm{~s}(\mathrm{SD}=5.61)$ for cubes and $6.08 \mathrm{~s}$ for spheres $(\mathrm{SD}=3.18)$. These completion times were larger than those reported for single view visual feedback $(4.26 \mathrm{~s}(\mathrm{SD}=1.93)$ for cubes and 3.48s $(\mathrm{SD}=1.55)$ for spheres). This was expected as adding a side view camera for dual visual feedback makes participants aware of their inaccuracy in grasp placement, and leads participants to spend more time adjusting their grasp for the purpose of achieving more grasp accuracy.

\subsubsection{Usability Analysis}

SUS average score for the different sizes test was $77(\mathrm{SD}=16.45) . \quad 592$

Out of 15 participants, $6(37.50 \%)$ preferred to look first to the frontal view while $8(53.33 \%)$ focused their attention on the side view first, one user remained undecided. To the question of which view was the most important for them, the opinion was divided into $7(46.66 \%)$ users referring to use the frontal view more, while the remaining 8 relied more on side view $(53.33 \%)$. With respect to which view was considered more important during the performance of the experiment, $7(46.66 \%)$ users considered it to be the frontal view while $7(46.66 \%)$ chose the side view. One user remained undecided. On using the system again, 9 users $(60.0 \%)$ will interact with the system again with dual visual feedback while 5 of the remaining $(33.33 \%)$ did prefer the single view interaction [AlKalbani et al. 2016]. 13 participants out of the 15 available had a specific approach for using dual visual feedback.

\section{Object Position Experiment}

We used a $2 \times 3 \times 3 \times 3$ within-subjects design, with two primary conditions: object position and object type (see Table 1). All new 15 participants took part in both conditions. Every permutation of position for both object types was randomly presented to participants to exclude potential learning effects. In total, each participant completed 27 (positions) $\times 2$ (objects) $=54$ trials and 810 grasps (54 trials $\times 15$ participants). Each static grasp of every participant was recorded for 5 seconds ( 75 frames), leading to collecting 60750 raw data points ( 810 grasps $\times 75$ frames).

Hypothesis: We test the null hypotheses that using dual visual feedback in grasping virtual objects that change in position has no effect on a) grasp aperture and b) grasp displacement.

\subsection{Procedure}

Participants were instructed to accurately locate and match their grasp aperture to the size and position of the virtual object in the shortest time possible on both feedback views. 27 different positions in all axes (x,y and $\mathrm{z}$ ) are used (see Table 1), covering a working range of $400 \mathrm{~mm}$ from participants (see Fig. 1b). We chose the object sizes that had the lowest mean difference between $G A p$ and object size found in literature [Al-Kalbani et al. 2016] (80mm for cubes and $70 \mathrm{~mm}$ for spheres).

During the experiment, an object (cube or sphere) appeared to participants on the feedback monitor, each object had 27 different positions. Size of both objects was unchanged throughout the experiment.

\subsection{Results and Analysis}

The object position that was used in the Object Size Experiment (Centre Middle) was changed in the $\mathrm{x}, \mathrm{y}$ and $\mathrm{z}$ axes (see Table. 1). We report on results of the $\mathrm{z}$ plane that was used in the Object Size Experiment $(1600 \mathrm{~mm})$, and changes in object positions were compared as whole sets between the two feedback methods (single and dual view visual feedback) to test the influence on visual feedback method on GAp and GDisp given that object position changes.

\subsubsection{Results - Grasp Aperture (GAp)}

Statistically significant difference was found in GAp in different positions between the two feedback methods in grasping cubes $\left(\chi^{2}(1)\right.$ $=647.99, p<0.01)$ and spheres $\left(\chi^{2}(1)=2508, p<0.01\right)$. A small effect size for cubes $(d>0.30$ and a medium effect size for spheres $(d>0.50)$ were found.

As shown in Fig. 5b, highest mean differences between $G A p$ and object size were in positions to the right hand side of participants (Top Right, Centre Right and Bottom Right) for cubes ( $M$ $=75.21 \mathrm{~mm}, \mathrm{SD}=15.29)$ and spheres $(M=57.06 \mathrm{~mm}, \mathrm{SD}=15.30)$. This contradicts results from the one visual feedback study as participants performed best in positions to their right hand side (see Fig. 5a), however, the opposite was present in dual view visual feedback. This is potentially attributed to the additional side view cam- 


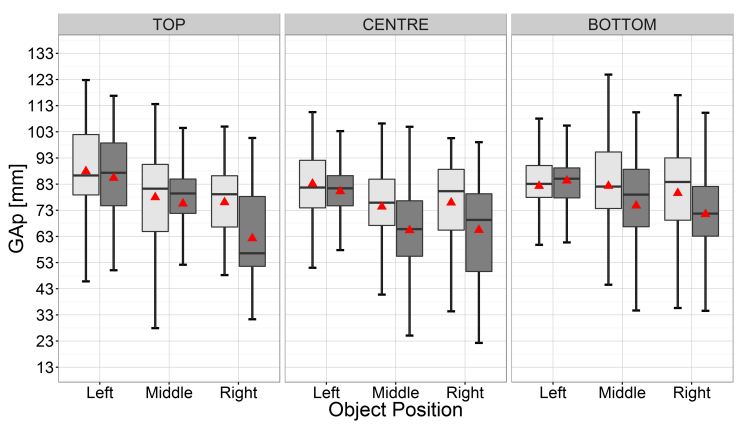

(a)

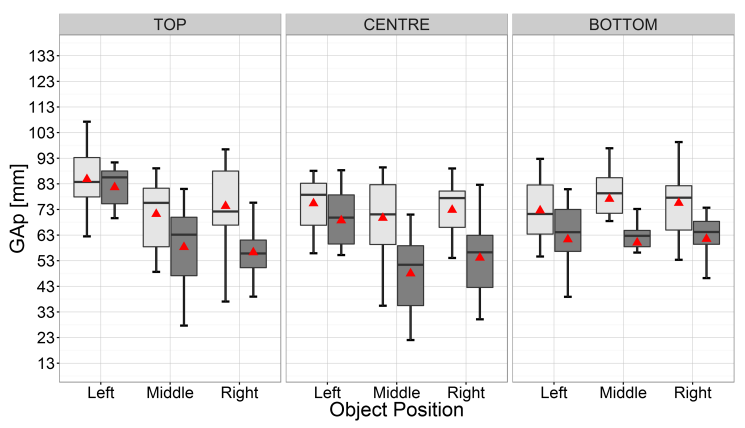

(b)

Figure 5: GAp for different positions in the $1600 \mathrm{~mm}$ z plane in the Object Position Experiment. Light grey boxplots show cubes, and dark grey boxplots show spheres. Red triangles on boxplots indicate the mean GAp across all participants for each size.

Whiskers represent the highest and lowest values within 1.5 times the interquartile range from the lower and upper quartiles: (a) Single view visual feedback from [Al-Kalbani et al. 2016] (b) Dual view visual feedback

and $73.87 \mathrm{~mm}(\mathrm{SD}=17.73)$ for spheres, thus participants performed better in matching their $G A p$ to object sizes using single view visual feedback. This can be explained by the fact that virtual objects changed position, and as participants had no prior knowledge about the positions of the virtual object that are presented in this experiment, accurately locating virtual objects in 3D space using their $g m p$ was prioritised over accurately match their GAp to object size. Even though this behaviour was present in single view visual feedback, presenting second view visual feedback to participants made this behaviour more prominent. As statistically significant results were found for the feedback method condition, the null hypothesis that dual visual feedback in grasping virtual objects that change in position does not have an effect on GAp is rejected.

\subsubsection{Results - Grasp Displacement (GDisp)}

Statistically significant difference was found GDis $p_{x}$ between the two feedback methods in grasping cubes $\left(\chi^{2}(1)=210, p<0.01\right)$ and spheres $\left(\chi^{2}(1)=23, p<0.01\right)$ in different positions. Negligible effects for cubes and spheres $(d<0.30)$ were found.

Mean GDis $p_{x}$ was lower for both objects across all positions using dual view visual feedback $(26.22 \mathrm{~mm}(\mathrm{SD}=22.54)$ for cubes and $24.32 \mathrm{~mm}(\mathrm{SD}=29.20)$ for spheres $)$ than single view visual feedback $(30.39 \mathrm{~mm}(\mathrm{SD}=26.90)$ for cubes and $27.55 \mathrm{~mm}(\mathrm{SD}=30.89)$ for spheres), thus using dual view visual feedback reduced GDis $p_{x}$ across all positions.
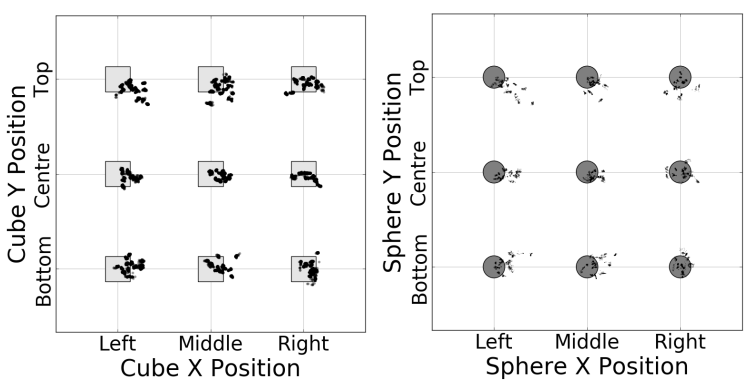

(a)
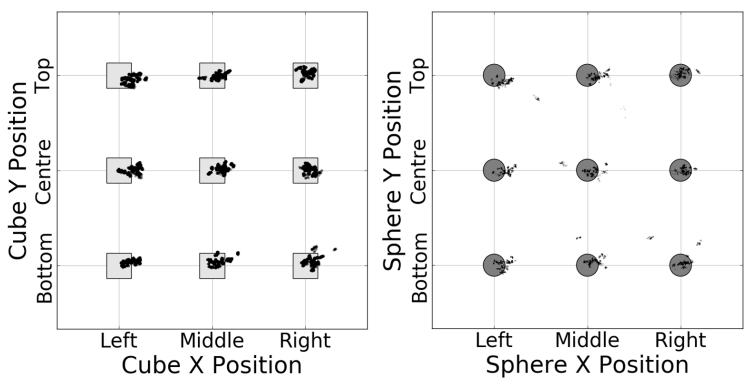

(b)

Figure 6: gmp placement (black clusters) in the $x$ and $y$ axes of all participants in the Object Position Experiment for cubes (left) spheres (right): (a) Single view visual feedback from [Al-Kalbani et al. 2016] (b) Dual view visual feedback size was found $(M=70.07 \mathrm{~mm}, \mathrm{SD}=18.60)$. This shows that participants tend to underestimate object size in the majority of positions when using dual view visual feedback. This was different for single view visual feedback where it was reported that consistent overestimation was present in positions to the left hand side of participants, and underestimation was consistent in positions to the right hand side of participants [Al-Kalbani et al. 2016]. Mean $G A p$ across all participants and positions was $60.91 \mathrm{~mm}(\mathrm{SD}=18.19)$ for cubes and $74.82 \mathrm{~mm}(\mathrm{SD}=14.60)$ for spheres, this shows that single view visual feedback outperforms dual view visual feedback 65 in $G A p$ matching to object size, as reported mean $G A p$ for single 654 view visual visual feedback was $79.94 \mathrm{~mm}(\mathrm{SD}=16.17)$ for cubes 655 Statistically significant difference was found in GDisp $p_{y}$ between the two feedback methods in grasping cubes $\left(\chi^{2}(1)=3026, p<\right.$ $0.01)$ and spheres $\left(\chi^{2}(1)=1349, p<0.01\right)$ in different positions. A medium effect for cubes $(d<0.80)$, and a small effect for spheres were found $(d<0.50)$. 
Mean GDispy across all positions was $4.21 \mathrm{~mm}(\mathrm{SD}=13.57)$ for 72 cubes and $0.29 \mathrm{~mm}(\mathrm{SD}=18.81)$ for spheres. GDisp $p_{y}$ values were 722 lower than those reported for one visual feedback $(-9.80 \mathrm{~mm}$ (SD $=723$ 21.99 ) for cubes and $-10.33 \mathrm{~mm}(\mathrm{SD}=24.13)$ for spheres). Inter- 724 estingly, positive mean GDisp y was found for both object across all 725 positions using dual view visual feedback, this contradicts findings 726 reported in one visual feedback, as GDisp y was found to be neg- 727 ative. This shows that participants in our study placed their $g m p \quad 728$ above the $o m p$ when interacting with virtual objects in a dual view ${ }_{729}$ visual feedback setup. Lower range of the mean GDisp $p_{y}$ across all 730 positions and objects was present using dual view visual feedback ${ }_{73}$ as shown in Fig. 6b, than single view visual feedback where a more 732 spread out range with higher deviation was reported for the mean 73

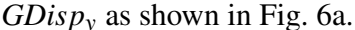

Our results show that dual view visual feedback outperforms single 735 view visual feedback by reducing mean GDis $p_{x}$ and GDisp $p_{y}$ across ${ }_{736}$ all positions which shifted placement of gmp towards the 0 origin ${ }_{737}$ of the $\mathrm{x}$ and $\mathrm{y}$ axis, and also by reducing the range of GDisp $p_{x}$ and ${ }_{738}$ GDisp $p_{y}$ with less deviation, meaning that participants were more 739 consistent in their spatial gmp placement in the $\mathrm{x}$ and $\mathrm{y}$ axes using dual view visual feedback.

Statistically significant difference was found in GDisp $p_{z}$ between the 741 two feedback methods in grasping cubes $\left(\chi^{2}(1)=2298, p<0.01\right)$ and spheres $\left(\chi^{2}(1)=1990, p<0.01\right)$ in different positions. Large 743 effects for cubes and spheres $(d>0.80)$ were found.

Similar to the Object Size Experiment, GDisp $p_{z}$ presented the high- 747 est displacement out of all three axis with single view visual feed- 748 back in [Al-Kalbani et al. 2016] for grasping virtual object in differ- 749 ent positions. Here mean GDisp $p_{z}$ across all positions was $3.33 \mathrm{~mm} 750$ $(\mathrm{SD}=22.17)$ for cubes and $5.07 \mathrm{~mm}(\mathrm{SD}=26.28)$ for spheres. This 75 shows a significant improvement in spatial gmp placement in the $\mathrm{z} 752$ axis as reported GDisp $z$ means for single view visual feedback were 753 $-58.75 \mathrm{~mm}(\mathrm{SD}=94.90)$ for cubes, and $-51.60 \mathrm{~mm}(\mathrm{SD}=89.06) 754$ for spheres. Moreover, lower deviation was shown with the use of dual view visual feedback, indicating that participants had less 75 variability in their depth estimation across all positions. Positive mean GDisp $p_{z}$ was present for both objects across all positions, this contradicts findings in [Al-Kalbani et al. 2016] where underestimation (negative GDisp ) of object position in the $\mathrm{z}$ axis was reported for both objects across all positions. This shows that dual view visual feedback led participants to overestimate object position in the $\mathrm{z}$ axis across all positions by placing their gmp in front of the omp. Mean GDisp $z$ was less spread and closer to the 0 origin on the $\mathrm{z}$ axis for both objects across all positions using dual view visual feedback as shown in Fig. 7b, in comparison to single view visual feedback (see Fig. 7a). As statistically significant results were found for the feedback method condition, the null hypothesis that dual visual feedback in grasping virtual objects that change in position does not have an effect on GDisp is rejected. As dual visual feedback has a significant effect on GDisp in all axes (x, y and z).

\subsubsection{Results - Completion Time}

Statistically significant difference across all positions in completion time between the two visual feedback methods was found for cubes $\left(\chi^{2}(1)=5778, p<0.01\right)$ and spheres $\left(\chi^{2}(1)=6212, p<0.01\right)$. Statistically significant difference in completion time between the two feedback methods shows that adding a side view camera for visual feedback significantly increases completion time, as the overall mean completion time across all positions and participants was $11.43 \mathrm{~s}(\mathrm{SD}=8.63)$ for cubes and 10.41s $(\mathrm{SD}=6.48)$ for spheres. These completion times were higher than those reported for single view visual feedback $(6.30 \mathrm{~s}(\mathrm{SD}=5.29)$ for cubes and $5.14 \mathrm{~s}(\mathrm{SD}=$ 2.63) for spheres).

Results from the Object Size and Object Position Experiments in this work have shown that dual view visual feedback makes a sig- nificant impact on spatial positioning in the $\mathrm{x}$ and $\mathrm{y}$ axes. Moreover, depth perception has improved in the $\mathrm{z}$ axis in both experiments in this study, as GDisp $z$ was significantly reduced, making grasping virtual objects in a single or varying position more accurate in terms of spatial positioning of a grasp. However, this study has also shown that adding a second view camera for visual feedback does not improve accuracy of $G A p$ matching to object size in comparison to single view visual feedback. Moreover, significantly higher completion time was also present in both experiments in this work meaning that even though more accuracy can be achieved in spatial positioning of gmp in all axes, completion time increases a result. This shows that a speed-accuracy trade-off must be made before utilising dual view visual feedback.

In addition, the grasp variation problem that was presented in one visual feedback (see Fig. 8a) is reduced using dual view visual feedback as shown in Fig. 8b. This shows that dual view visual feedback allows participants to visualise their whole hands using frontal and side visual feedback eliminates the need to adapt grasp type, thus causing less grasp variation.

\subsubsection{Usability Analysis}

SUS average score for the different sizes test was $64.5(\mathrm{SD}=13.43)$. Out of 15 participants, $8(53.3 \%)$ referred to look first to the frontal view while $6(37.5 \%)$ focused their attention on the side view first, one user remained undecided. To the question of which view was the most important for them, the opinion was divided into $9(60.0 \%)$ users preferring to use the frontal view more, while 5 relied more on side view $(33.33 \%)$. With respect to which view was considered more important during the performance of the experiment, $11(73.33 \%)$ users considered it to be the frontal view while 4 $(26.66 \%)$ chose the side view. On using the system again, 12 users $(80.0 \%)$ will interact with the system again with dual view visual feedback while $2(13.33 \%)$ did preferred single view interaction ([Al-Kalbani et al. 2016]). 12 participants out of the 15 available had a specific approach for using dual visual feedback.

\section{Conclusions And Future Work}

This work presented a first study into the use of dual view visual feedback in an exocentric MR environment for assisting freehand grasping of virtual objects. Measures of Grasp Aperture $(G A p)$ and Grasp Displacement (GDisp $p_{\mathrm{xyz}}$ ) were used to quantify grasp ability and comparisons given against traditional single view visual feedback. We presented a comprehensive study of the dual view visual feedback focusing on mitigating the problems found in freehand grasping of virtual objects in an exocentric MR environment, namely grasp displacement in the $\mathrm{x}, \mathrm{y}$ and axes (GDisp $\left.p_{\mathrm{xy}}\right)$, high displacement in the $\mathrm{z}$ axis $\left(G D i s p_{z}\right)$ and inaccurate object size estimation using GAp.

The results illustrate that dual view visual feedback significantly improves Grasp Displacement in the $\mathrm{x}$ and y axes $\left(G D i s p_{\mathrm{xy}}\right)$. Furthermore user estimation of the object $\mathrm{z}$ position (the highest displacement found in the single view study) was significantly improved with the dual view feedback over single view feedback. This mitigation of displacement in the $\mathrm{z}$ axis was attributed to users increased awareness of their placement errors in the $\mathrm{z}$ axis via the additional side view feedback, thus allowing them to correct their grasp placement (see Table 2).

We also illustrate similarities between the two feedback methods (single view and dual view) in user estimation of object size using $G A p$. With single view feedback outperforming dual view visual feedback in matching $G A p$ to object size. In the object position experiment participants were more focused on position change over object size, thus similar to single view feedback, $G A p$ varies less than expected using dual visual feedback, and was not proportional to object size. These findings are important when understanding how users respond to different visual feedback views and notewor- 
Table 2: Descriptive Statistis (Mean \pm SD): Summary statistics of single [Al-Kalbani et al. 2016] and dual visual feedback: all mean/SD values are calculated across all participants, object sizes and object types for the Object Size experiment, and across all participants, object positions and object types for the Object Position experiment

\begin{tabular}{c|c|c|c|c|c|c|c}
\hline \hline Experiment & Feedback & $\boldsymbol{G A p}[\mathbf{m m}]$ & $\boldsymbol{G D i s}_{\boldsymbol{x}}[\mathbf{m m}]$ & $\boldsymbol{G D i s} \boldsymbol{p}_{\boldsymbol{y}}[\mathbf{m m}]$ & $\boldsymbol{G D i s}_{\boldsymbol{z}}[\mathbf{m m}]$ & Time $[\mathbf{s}]$ & SUS Score \\
\hline \multirow{2}{*}{ Object Size } & Dual View & $78.55 \pm 31.28$ & $25.17 \pm 21.65$ & $-8.86 \pm 12.24$ & $-3.88 \pm 25.69$ & $6.87 \pm 4.63$ & $77 \pm 16.45$ \\
\cline { 2 - 8 } & Single View & $73.43 \pm 27.65$ & $29.77 \pm 14.49$ & $-11.10 \pm 12.30$ & $-34.12 \pm 61.24$ & $3.87 \pm 1.80$ & \\
\hline \multirow{2}{*}{ Object Position } & Dual View & $70.17 \pm 20.04$ & $27.78 \pm 31.76$ & $0.62 \pm 18.71$ & $4.91 \pm 33.42$ & $11.22 \pm 7.79$ & $64.50 \pm 13.43$ \\
\cline { 2 - 8 } & Single View & $76.72 \pm 19.16$ & $27.82 \pm 31.13$ & $-10.75 \pm 28.35$ & $-63.70 \pm 116.95$ & $6.02 \pm 4.46$ & \\
\hline \hline
\end{tabular}
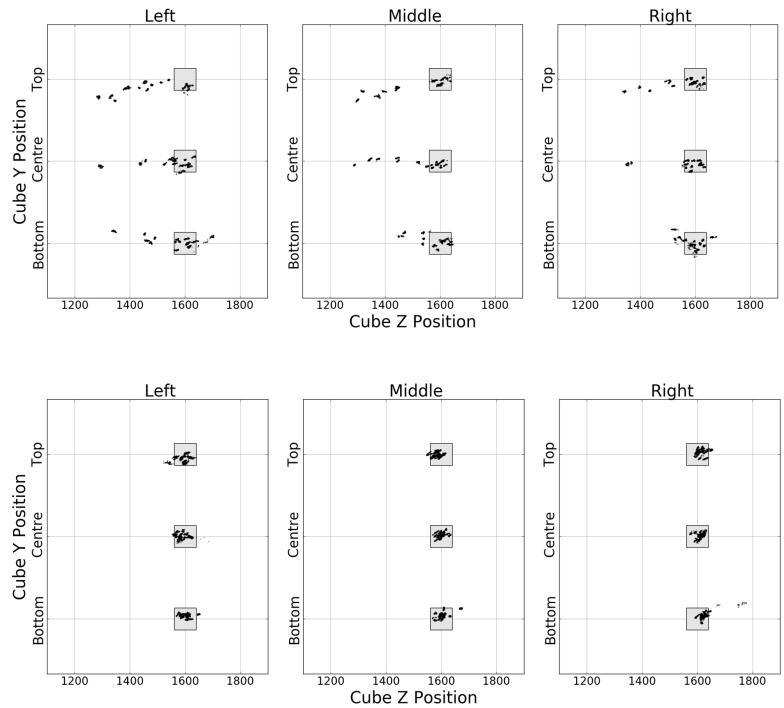

(a)
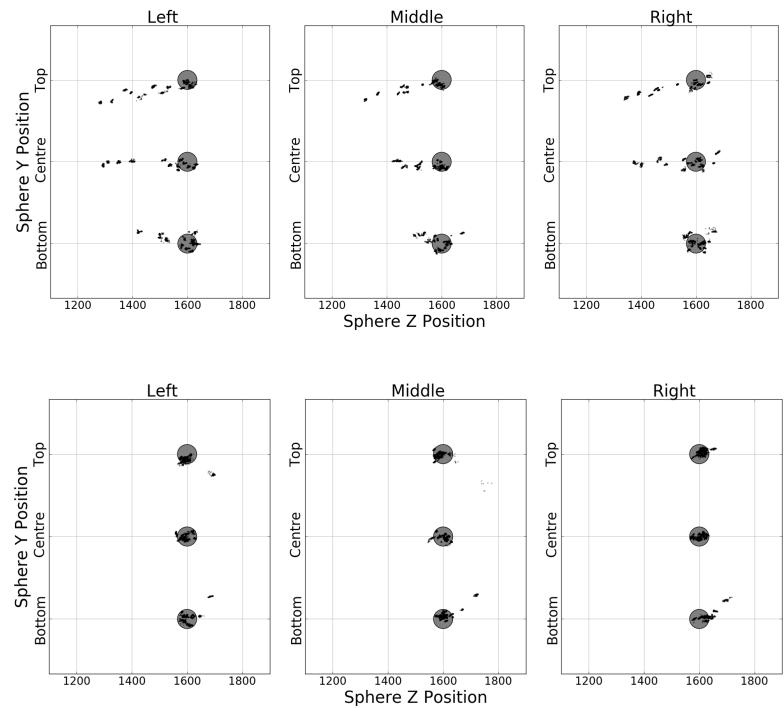

(b)

Figure 7: gmp placement (black clusters) in the z axis of all participants in the Object Position Experiment for cubes (left) spheres (right): (a) Single view visual feedback from [Al-Kalbani et al. 2016] (b) Dual view visual feedback
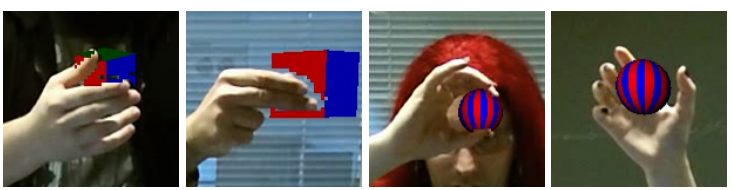

(a)
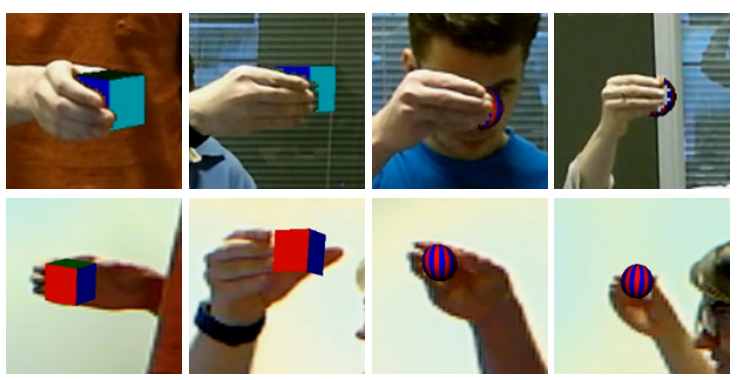

(b)

Figure 8: Examples of the difference in grasp variation shown by participants in grasping cubes and spheres in the same positions in 3D space between the two feedback methods: (a) Single view visual feedback from [Al-Kalbani et al. 2016] (b) Dual view visual feedback (where top row shows the frontal view, and bottom row shows the side view)

thy for future work developing freehand grasping systems. Our work also shows that changing the visual feedback method does not improve size estimation using $G A p$, as it remains within a mean range of $60 \mathrm{~mm}(\mathrm{SD}=31.28)$ to $70 \mathrm{~mm}(\mathrm{SD}=31.09)$ across all participants and object types regardless of changes in object size and the feedback method employed.

We further report that completion time significantly increases using dual view visual feedback, thus even though our proposed feedback method significantly improves spatial grasp placement, it results in longer completion times (see Table 2). This is attributed to participants repeatedly correcting their grasp posture for either aperture or position using the additional side view visual feedback. This increase in completion time should be considered in future interaction design, however in our work performance was task dependant and grasp accuracy was the primary goal. In addition, grasp variation that was present using single view visual feedback, was reduced using dual view visual feedback. This indicates that enabling participants to visualise their hands using side and front views encourages more consistency in the grasp type.

Finally, from the usability analysis we can draw the following conclusions: According to the SUS ranking system of Bangor et al. [2009] the dual view visual feedback was rated as "good and acceptable" with a score 77 (SD = 16.45) for the object size experiment, while it was rated as "OK and marginally acceptable" for the object position experiment with a score of 64.5 (SD = 13.43) [Bangor et al. 2009] (see Table 2). According to this, when the object position in the MR space changes for every test iteration participants found the use of dual view visual feedback more challenging. Finally, although there was a divided opinion in both experiments about which view is the most important, the majority of users concluded that they will interact again with the dual view visual feed- 
back method, and consider this method more accurate and helpful 88 for locating virtual objects in an MR environment. Future work will 882 consider the changes to grasp accuracy when using different posi- 883 tions of the dual view visual feedback in this exocentric MR setting, 884 alongside the integration and translation of this form of feedback to 885 optical see-through AR/MR systems.

\section{References}

AL-KALBANI, M., WILLIAMS, I., AND MAITE, F.-P. 2016. Analysis of Medium Wrap Freehand Virtual Object Grasping in 890 Exocentric Mixed Reality. IEEE ISMAR, accepted in press.

BAngor, A., Kortum, P., AND Miller, J. 2009. Determin- 892 ing what individual sus scores mean: Adding an adjective rating 893 scale. Journal of usability studies 4, 3, 114-123.

Baran, M., Lehrer, N., Siwiak, D., Chen, Y., Duff, M., 895 INGALLS, T., AND RIKAKIS, T. 2011. Design of a home-based 896 adaptive mixed reality rehabilitation system for stroke survivors. ${ }^{897}$ In IEEE EMBC, IEEE, 7602-7605.

Benko, H., Wilson, A. D., And Zannier, F. 2014. Dyadic 899 projected spatial augmented reality. In ACM UIST, 645-655. 900

BROOKE, J. 1996. Sus-a quick and dirty usability scale. Usability 90 evaluation in industry 189, 194, 4-7.

Bullock, I. M., FeiX, T., AND Dollar, A. M. 2013. Finding 90 small, versatile sets of human grasps to span common objects. IEEE ICRA, 1068-1075.

Chang, C.-Y., Lange, B., Zhang, M., Koenig, S., Re- 900 Quejo, P., Somboon, N., SAwChuK, A. A., AND RIzzo, 907 A. A. 2012. Towards pervasive physical rehabilitation using mi- 908 crosoft kinect. In 6th PervasiveHealth Conference, IEEE, 159- 909 162.

CoHen, J. 1992. A power primer. Psychological Bulletin 112, 1, 91 $155-159$.

Coles, T. R., John, N. W., Gould, D. A., ANd CALDwell, 913 D. G. 2011. Integrating haptics with augmented reality in a 914 femoral palpation and needle insertion training simulation. Hap- 915 tics, IEEE Transactions on 4, 3, 199-209.

DEY, A., AND SANDOR, C. 2014. Lessons learned: Evaluating 917 visualizations for occluded objects in handheld augmented real- 918 ity. International Journal of Human-Computer Studies 72, 10, 919 704-716.

Dey, A., Cunningham, A., AND SAndor, C. 2010. Evaluating 921 depth perception of photorealistic mixed reality visualizations 922 for occluded objects in outdoor environments. In ACM VRST, ${ }_{923}$ 211-218.

Duff, M., Chen, Y., Attygalle, S., Herman, J., Sun- 925 DARAM, H., QIAN, G., He, J., AND RiKAKIS, T. 2010. An ${ }^{926}$ adaptive mixed reality training system for stroke rehabilitation. ${ }^{92}$ IEEE Transactions on Neural Systems and Rehabilitation Engi- 92 neering 18, 5, 531-541.

Duff, M., Chen, Y., ATtygalle, S., Sundaram, H., And 930 RIKAKIS, T. 2010. Mixed reality rehabilitation for stroke ${ }^{93}$ survivors promotes generalized motor improvements. In IEEE 932 $E M B C, 5899-5902$.

DunN, O. J. 1961. Multiple Comparisons Among Means. Journal ${ }^{934}$ of the American Statistical Association 56, 293, 52-64.

FeiX, T., Bullock, I. M., AND Dollar, A. M. 2014. Analysis 936 of Human Grasping Behavior: Correlating Tasks, Objects and ${ }_{937}$ Grasps. IEEE Transactions on Haptics 7, 3, 311-323.

FIELD, A. 2012. Discovering statistics using $R$. Sage publications. 939 HA, T., FEIner, S., AND Woo, W. 2014. Wearhand: Head- 940 worn, rgb-d camera-based, bare-hand user interface with visually ${ }^{941}$ enhanced depth perception. In IEEE ISMAR, IEEE, 219-228.

Hilliges, O., KIM, D., IZADI, S., Weiss, M., AND WILSON, ${ }^{943}$ A. 2012. Holodesk: direct 3d interactions with a situated see- 944 through display. In Proceedings - SIGCHI Conference on Human 945 Factors in Computing Systems, ACM, 2421-2430.
Horeman, T., Rodrigues, S. P., VAn den Dobbelsteen, J. J., Jansen, F.-W., AND DAnKelman, J. 2012. Visual force feedback in laparoscopic training. Surgical endoscopy 26, $1,242-248$.

IMAN, R. L., AND J., C. W. 1983. Multiple comparisons procedures based on the rank transformation. Proceedings - Joint Statistical Meetings, Houston Texas, August.

Jia, D., Bhatti, A., Nahavandi, S., AND Horan, B. 2013. Human performance measures for interactive hapticaudio-visual interfaces. Haptics, IEEE Transactions on 6, 1, 46-57.

Johnsen, K., Ahn, S. J., Moore, J., Brown, S., RobertSON, T. P., MARABLE, A., AND BASU, A. 2014. Mixed reality virtual pets to reduce childhood obesity. Visualization and Computer Graphics, IEEE Transactions on 20, 4, 523-530.

Jones, B., Sodhi, R., Murdock, M., Mehra, R., Benko, H., Wilson, A., Ofek, E., Macintyre, B., RaghuvanSHI, N., AND ShapIRA, L. 2014. Roomalive: Magical experiences enabled by scalable, adaptive projector-camera units. In ACM UIST, 637-644.

KRUSKAL, W. H., AND WALLIS, W. A. 1952. Use of ranks in onecriterion variance analysis. Journal of the American statistical Association 47, 260, 583-621.

Lee, J., Olwal, A., IshiI, H., And Boulanger, C. 2013. Spacetop: integrating $2 \mathrm{~d}$ and spatial $3 \mathrm{~d}$ interactions in a seethrough desktop environment. In Proceedings - SIGCHI Conference on Human Factors in Computing Systems, ACM, 189-192.

MaCKenZIE, C. L., AND IBERALL, T. 1994. The grasping hand, vol. 104. Elsevier.

Maria, K., FilipPeschi, A., RufFaldi, E., ShORR, Y., AND GOPHER, D. 2015. Evaluation of multimodal feedback effects on the time-course of motor learning in multimodal vr platform for rowing training. In IEEE ICVR, 158-159.

OLDFIELD, R. 1971. The assessment and analysis of handedness: the Edinburgh inventory. Neuropsychologia 9, 1, 97-113.

Pacchierotti, C., Chinello, F., Malvezzi, M., Meli, L., AND PratTiChIzZO, D. 2012. Two finger grasping simulation with cutaneous and kinesthetic force feedback. In Haptics: perception, devices, mobility, and communication. Springer, 373382.

Pitts, M. J., Burnett, G., Skrypchuk, L., Wellings, T., AtTridge, A., AND Williams, M. A. 2012. Visual-haptic feedback interaction in automotive touchscreens. Displays 33, 1 , 7-16.

PRACHYABRUED, M., AND Borst, C. W. 2014. Visual feedback for virtual grasping. In IEEE 3DUI, 19-26.

Prattichizzo, D., PACChierotti, C., AND Rosati, G. 2012. Cutaneous force feedback as a sensory subtraction technique in haptics. Haptics, IEEE Transactions on 5, 4, 289-300.

RÉBillat, M., Boutillon, X., Corteel, É., AND Katz, B. F. 2012. Audio, visual, and audio-visual egocentric distance perception by moving subjects in virtual environments. ACM Transactions on Applied Perception 9, 4, 19.

Sutherland, C., Hashtrudi-ZaAd, K., Sellens, R. Abolmaesumi, P., AND Mousavi, P. 2013. An augmented reality haptic training simulator for spinal needle procedures. Biomedical Engineering, IEEE Transactions on 60, 11, 30093018.

Vieira, J., Sousa, M., Arsénio, A., And Jorge, J. 2015. Augmented reality for rehabilitation using multimodal feedback. In Proceedings - Workshop on ICTs for improving Patients Rehabilitation Research Techniques, ACM, 38-41.

Wilson, A. D., And Benko, H. 2010. Combining multiple depth cameras and projectors for interactions on, above and between surfaces. In ACM UIST, 273-282. 